\title{
"Going the extra mile": A descriptive exploratory study of Primary Health Services based on the experiences of Pacific Primary Health Organisation Service managers and providers
}

Margaret Pack, Private practice, New Zealand

\begin{abstract}
INTRODUCTION: This exploratory study is part of a larger evaluation of the primary health care strategy (PHCS) in Aotearoa New Zealand, using a mixed methods research approach. The aims of this qualitative arm of the research were to explore the extent of use and satisfaction with the PHCS through the operation of Pacific-led Primary Health Organisations (PHOs) in relation to service provision and delivery from the service providers' and managers' perspectives.

METHOD: The exploratory study was conducted using a case study design and in-depth interviews with service managers and health providers at six Pacific-led PHOs. A review of the literature on primary healthcare was conducted prior to undertaking the research. In this literature review, several themes were noted from the review of policy documents providing background to the development of primary healthcare in New Zealand.

CONCLUSION: The themes from interviews suggest a core tension between the business model, Ministry reporting requirements, and more altruistic values of both managers and service providers in their delivery of services. Overall, there was a positive response to the lowered cost of healthcare from the providers and managers interviewed in the Pacificled primary health services, mirroring the findings of the larger evaluation report of PHOs (Cumming et al., 2005). The availability of wrap-around, holistically based, accessible services delivered by culturally responsive health providers who were considered to "go the extra mile" for their clients was the predominant theme accounting for an increased uptake and use of the services. The implications for health social work are discussed.
\end{abstract}

KEYWORDS: primary health, mental health, social work and Pacific health

\section{Introduction}

This article begins by providing the demographic and historical context in which the Pacific-led PHOs were developed in Aotearoa New Zealand from the mid-2000s. Definitions of the terms Pacific; and Pasifika are given and some of the key barriers in the provision and uptake of services are then outlined. The literature review underpinning the research is reported, along with the aims and objectives of the project and its research design and methods. Finally, the results of the data analysis are summarised and the
AOTEAROA

NEW ZEALAND SOCIAL WORK 30(2), 57-67.

CORRESPONDENCE TO: Margaret Pack margaret.pack@slingshot.co.nz 
article concludes with key implications and recommendations for health social work in Aotearoa New Zealand.

\section{Definitions and demographic trends}

Pacific peoples within the context of the present study is an umbrella term used to describe those residents and citizens living in Aotearoa New Zealand who self-identify culturally with one or more of the predominant Pacific cultures living there. The predominant Pacific cultures represented in the present Aotearoa New Zealand population include: Samoan, Tokelau Islander, Cook Island Māori, Niuean, Tongan and Fijian. The term Pasifika relates to those born in Aotearoa New Zealand with a Pacific heritage which has been used to distinguish those residents from those born in the Pacific Islands who later migrated to live in Aotearoa New Zealand. The current research on the Pacific-led PHOs encompasses both groups, whom together comprise the term Pacific peoples, or those who identify culturally with one or more of the Pacific cultures represented in Aotearoa New Zealand, regardless of place of birth.

In 2013, the Pacific ethnicity with the highest proportion of Aotearoa New Zealand-born people included those self-identifying as Niuean, with $78.9 \%$ born in Aotearoa New Zealand.Those self-identifying as Cook Island Māori were $77.4 \%$ of the Aotearoa New Zealand population; Tokelauan $73.9 \%$; Samoan, 62.7\%; and Tongan 59.8\% (Statistics New Zealand, 2013). In 2013, 7.4\% of the population (295,941 people) identified with one or more Pacific ethnic groups, compared with $6.9 \%$ (265,974 people) in 2006. However, the rate of growth for the Pacific peoples ethnic group slowed across recent censuses, growing 14.7\% between 2001 and 2006 but only 11.3\% between 2006 and 2013. The Pacific peoples ethnic groups whose growth slowed between 2006 and 2013 included Tongan, Samoan, Cook Island Māori, Niuean and Tokelauan. In contrast, the Fijian ethnic group grew by a bigger percentage between
2006 and 2013 (46.5\%) than between 2001 and 2006 (40.1\%) (Statistics New Zealand, 2013).

\section{Literature review: Barriers to Pacific healthcare}

The following section summarises the predominant themes found in the literature review which framed the research. These themes include the barriers faced by Pacific peoples to accessing and using healthcare in Aotearoa New Zealand; the prevalence of long-term health conditions and low uptake of health services amongst this population; and the evolution of the PHO service network aiming to ameliorate the barriers to accessing and using healthcare by Pacific peoples. Removing the business imperative from healthcare that enables innovation including a culturally appropriate healthcare model is the predominant theme of the literature review.

There are many barriers that have been identified involving Pacific peoples' access and use of health care in Aotearoa New Zealand. Pacific peoples are disproportionately represented in the most deprived areas of the country and have poorer health status than other New Zealanders (Pack, Minister, Churchward, \& Fa'asalele Tanuvasa, 2013). Thus, Pacific citizens and residents in New Zealand are a key priority group for the primary health services, given the focus on reducing inequalities in health. The PHCS was implemented by the Labour Government in Aotearoa New Zealand in the mid-2000s. The services established were evaluated to determine what the impact was on the delivery of primary health care services nationally and the resulting changes in the health of local geographic populations of enrolled residents. The PHCS had a focus on services for Pacific peoples provided by Pacific peoples, active involvement of Pacific communities in service delivery, further building of Pacific provider capacity, the formation of Pacific-led services, and leadership at a national level. All providers of PHOs were to identify, reach out to and address Pacific health needs (King, 2001). 
The key intention of the PHCS is the removal of the business emphasis in primary health thus opening the way to the development of culturally relevant models of healthcare provision and delivery. Re-structuring care teams to include allied health beyond simply nursing and medical staff; delivering education to patients on how to manage healthcare and using Pacific languages in healthcare delivery are some of the ways suggested to overcome barriers to addressing Pacific health needs (Beddoe \& Deeney, 2012; Döbl, Beddoe, \& Huggard, 2017; Keating \& Jaine, 2016; Southwick, Kenealy, \& Ryan, 2012).

\section{Accessibility of services and long-term health conditions}

Concerns about the accessibility of health care, influenced by increases in the prevalence of chronic conditions and an ageing provider workforce have dominated the literature on primary health service evaluations worldwide (Hogg, Rowan, Russell, Geneau, \& Muldoon, 2008). Recent frameworks for primary healthcare internationally have emphasised the service delivery aspects guided by principles of "comprehensiveness, integration and accessibility" (Hogg et al., 2008, p. 308). In the Canadian and Aotearoa New Zealand contexts, indigenous populations have been consulted and new models of healthcare provision have thus developed. These models are designed to tackle the social determinants of health which inevitably impact, relative to poorer health outcomes and lower life expectancy than for European service users (Barnett \& Barnett, 2004). The context of historical colonisation has been cited as influencing equity and as a social determinant of health among Pacific nations (Anderson et al., 2006). Under the Treaty of Waitangi, the founding charter between Māori and Pākehā in Aotearoa New Zealand, partnership, participation and protection are guiding principles, which necessitate a focus on identifying and addressing inequities in health as in other areas of life (Anderson et al., 2006). Social work in Aotearoa

New Zealand has enshrined in its professional standards of practice, standards aimed at working for greater equity under the Treaty, encompassing all areas of service provision including health (Beddoe \& Deeney, 2012; Döbl et al., 2017; Pockett \& Beddoe, 2017). Barriers to accessing primary health care in Aotearoa New Zealand continue to revolve around the financial cost of seeing a general practitioner, with the survival strategies of service users including delaying seeking care, lack of uptake of medication and putting others in the family first, such as children and the elderly (Barnett \& Barnett, 2004; Hawley \& McGarvey, 2015; PulotuEndemann \& Faleafa, 2017).

Alongside these principles underpinning health models, the broader focus in primary healthcare has been on community empowerment, education and the demographic and cultural aspects of health (Hogg et al., 2008). Western models of health care involving diagnosis and treatment often do not conform to the cultural norms of Pacific service users and their aiga (family) and the wider $n u^{\prime} u$ (village, community). Pacific models of healthcare to address these differences need to integrate principles of choice, self-determination, and culturally relevant models of health care delivery. This goal has been achieved in the field of mental health care by translating health information into Pacific languages, providing choices of provider, a range of support services and integrating hospitality as part of the care (Agnew et al., 2004; Pulotu-Endemann \& Faleafa , 2017; Southwick, Kenealy, \& Ryan, 2012; Suaalii-Sauni et al., 2009; Tamasese, Peteru, Waldegrave, \& Bush, 2005).

\section{Primary health care in Aotearoa New Zealand}

In relation to the Primary Health Care Services history, in February 2001, the New Zealand government released the Primary Health Care Strategy (PHCS) with the aim of improving the health of New Zealanders and reducing health inequalities. The five to 10 -year vision of the strategy was to shift primary health care 
(PHC) services to focus more on the health of the population by providing services which are easy to access; improving and maintaining their health; and coordinating their on-going care (King, 2001). Underlying this vision was a greater emphasis on the role of community participation in health improvement. PHC was seen to encompass a wide variety of services, including health promotion and preventive care, which necessitated the involvement of a wide range of health professionals (multidisciplinary teams) in the service delivery model.

To achieve the vision, the strategy emphasised six key directions for the future development of PHC in Aotearoa New Zealand: 1) work with local communities and geographic populations of enrolled residents; 2) identify and remove health inequalities; 3 ) offer access to comprehensive services to improve, maintain and restore people's health; 4) coordinate care across service areas; 5) develop the primary health workforce; 6) and continuously improve quality using good information (King, 2001).

A large number of PHOs were established between 2002 and 2005 whose brief was to address these aims. By mid-2008 there were 80 PHOs in operation, with additional funding to the value of $\$ 2.2$ billion having been provided for further PHC service developments since 2001 (Cumming \& Mays, 2011). Early evaluations have noted the unique way in which each $\mathrm{PHO}$ has been adapted to the communities in which they have developed. The dilemma is the struggle for smaller and remote PHOs to stay local when there have been pressures to amalgamate with larger PHOs to effect economies of scale (Gauld \& Mays, 2006). These amalgamations lead to a dilemma over control of services trying to remain relevant to local resident populations whilst maintaining altruism over a concern to show a profit (Gauld \& Mays, 2006).

The contribution of social workers to establishing PHO services based on social justice principles within these evaluations have indicated a synergy between social work and primary health care aims. Both aims are ideally structured and delivered by adhering to culturally relevant principles that acknowledge the holistic nature of health which includes the role of spirituality, community and family participation in healthcare (Pack, 2008). Jantrana and Crampton (2009) found that ethnicity and gender were significantly associated with higher odds of deferring buying a prescription. The low uptake of dental care due to high cost was identified as a compounding factor in the escalation of physical health problems including exacerbations of chronic conditions. Social workers, through advocating for a holistic vision of health, are ideally placed to highlight where barriers in health exist (Beddoe \& Deeney, 2012; Döbl et al., 2017; Pockett \& Beddoe 2017). Social work is well placed to suggest alternative models of health care.

This comprehensive, holistic, model acknowledges the importance of four facets of primary care service delivery that is prefaced on the importance of the patient and treatment provider relationship, awareness of the whole person, and gender, culture and family (Hogg et al., 2008). To evaluate the model, provider satisfaction is considered pivotal as treatment providers, when satisfied with the services they are working within, are found to be more open to alternative processes and a holistic and individually tailored approach when working in primary healthcare (Hogg et al., 2008).

\section{Method}

\section{Research aims}

The two main aims of the exploratory study were: 1) to identify the environmental and organisational context that impacted treatment providers and service managers of the PHOs; and 2) to identify the structural aspects of the policy and governance of the practice agency and its impact on the delivery of services by the provider, and, therefore, its impact on health outcomes. 
In undertaking this exploratory study, our research team comprising four Pacific health researchers, had earlier completed the interviews and transcribed the audio recordings. The author was then invited to analyse the data, report the major themes from the interviews, and to develop recommendations that were to sit alongside the larger mixed methods study on Pacific patients and their families' perspectives of the same PHOs. As I had been involved earlier in the establishment, development and service management of a culturally led PHO that was not part of the research, the team requested my involvement as they valued my background to provide rich and in-depth knowledge of the field of PHO development. The overarching study received Research Ethics Approval from Victoria University of Wellington's Research Ethics Committee.

\section{Research design and methodology}

An exploratory, descriptive, qualitative research design and methodology were used to explore the service managers and provider's perspectives of the structure and the day-to-day operation of their PHOs. The researchers adopted a case study approach based on Yin's description of case study (Yin, 2009). Each PHO was considered to be an example or a case, in the sense that each $\mathrm{PHO}$ had developed uniquely relative to its management structure/governance, service establishment and delivery due to a range of factors such as geography, size of resident population, funding or budget and local health demographics. Yin (2009) discusses the importance of triangulation in case study research for its potential to assemble different narrations on a theme. Thus we were able, in the current study, to incorporate service managers' views to explore how the service setup and structure impacted on the service delivery from a health provider's perspective within each PHO. A case study research approach enabled the context and structure of each $\mathrm{PHO}$ to be described alongside the accounts of health providers/managers and brought together with the service user accounts in the broader research project.

\section{Findings}

The following section presents the themes from the interviews conducted with managers and service providers. These themes were related to: 1) lowered costs of healthcare; 2 ) publicising the availability of services offered; 3 ) access to a range of services; 4 ) an ethic of care and "going the extra mile" for clients; 5) holistically based/integrated models of care; 6) incorporating culturally appropriate models of well-being; 7) relationship with community: PHO partnerships with NGOs, residents and local communities: 8) building workforce capability, and 9) providing services in a shared language. Due to the differing perspectives of the groups of participants, some themes were more figural for one group, for example the managers, than for service providers. In some themes both groups were in agreement about the issues. Therefore, in some themes, managers' perspectives predominated while in others, service providers' views did.

\section{Lowered costs of healthcare}

There was an enthusiastic response to the lowered cost of healthcare from the stakeholders interviewed. Reducing the costs of medical consultations was a primary motivation for practices to initially become involved in the PHOs. As one manager of a Pacific-led $\mathrm{PHO}$ explained during the first round of interviews in 2005:

...You know the first benefit to us is no or low cost and they [patients] don't pay. (Manager)

The CEO from another Pacific PHO stated in 2009 that the fee-paying structure still offered a means of providing a targeted approach to those patients most in need of low or no-cost consultations.

PHO I think it's a success with 90 plus percent enrolment throughout the 
country plus Pacific people, people are saying that they [lowered costs] are the advantage of PHOs. Low cost consultation fees I think is the main product ... So a lot of people have enrolled and are making use of the services. (Manager/CEO)

\section{Publicising the availability of low-cost services}

The availability of low-cost health care was not, however, widely known in the local community initially, which necessitated promotion of the PHO service. There was also a need to publicise the specific services that were offered. Information dissemination about how patients could enrol themselves and their family members in order to obtain access to low- or no-cost consultations, lower prescription fees and other services, was part of the implementation strategy of each of the PHO managers interviewed. For example, the use of promotional campaigns on Pacific Radio spoken in a range of Pacific languages was one way of publicising the availability to an audience of Pacific clients that was discussed at interview with one urban Pacific-led PHO. Community meetings with local groups were another way in which this $\mathrm{PHO}$ publicised the range of services their organisation offered. Fono organised by this PHO provided an opportunity to distribute more general information about health promotion to a range of audiences in faceto-face mode. It was considered important to follow up any presentations to answer queries and to hold meetings with the professional groups working at the PHO:

We have a very strong Samoan Residents' Association and we tell them about health stuff and the PHO as well and then we also have a meeting of other nurses of different communities and we tell them about the PHO. (Manager)

\section{Access to a range of services}

Access to a range of other services such as free transport to treatment and lower prescription costs were important incentives to establishing a PHO. This widespread appeal was seen by participants as a means of improving access to comprehensive health care services for residents. Another PHO organised health days to introduce a range of health services to local residents including their promoting their own services:

... we just have a health day, we go to a hall and stakeholders are invited to come and display their information and tell people about the services that they provide. (Manager)

\section{An ethic of care and "going the extra mile" for clients}

The attitudes of Pacific PHO staff towards their work were reported by participants to differ from the business orientation of many medical practices which worked from a business-centred model. This difference in philosophy was thought to partly stem from the values underpinning $\mathrm{PHOs}$ being supported by charitable trusts. A workplace based in a shared enthusiasm for helping under-resourced communities was the major motivation described by one general practitioner working in a $\mathrm{PHO}$ where $97 \%$ of the local enrolled population is described as "low income and Pacific Island":
The philosophy of this practice is improved access with lower fees ... affordability has always been an important part of the organisation really for us and for other members of the PHO... We provide a free taxi service for people who can't get to their appointments as well. We have access to free PHO funded prescriptions. (General practitioner)

Altruism, and the not-for-profit motivation to remain working within the $\mathrm{PHO}$ was seen by PHO health workers as important for putting funds back into community, as the same general practitioner interviewed suggested:

I'm a salaried GP so I don't get the financial incentives, it's not my business 
that I'm safeguarding, that's a different model from the sort of third sector where there's a long history of community ownership and not-for-profit being part of the way that we operate. (General practitioner)

\section{Holistically based /integrated models of care}

A community model of care facilitated by the PHOs was described as a positive development across the providers interviewed. This model consisted of several elements - remaining small enough to know the local community which enabled treatment providers to remain aware and responsive to locally defined needs. Coordination of services and communication across practices meant that duplication of services in a geographic area could be avoided as the following excerpt from an interview with a general practitioner in a Pacific-led PHO illustrates:

You know it's good to have that sort of relationship because of referral - we're basically seeing people from the same community. It helps avoid duplication of services and knowing what people are doing, having input with different families without knowing that each other is involved in, which I think happened a lot more under pre-PHOs. (General practitioner)

\section{Incorporating culturally appropriate models of well-being}

Pacific PHOs looked at health more holistically deriving from social inequalities and so they actively advocated on behalf of patients. A nurse described advocating with income support agencies on behalf of sickness and invalid beneficiaries who could not afford to see a general practitioner for review of their medical condition to avoid a cessation of weekly income benefit payments. She encouraged those patients with long-term or complex presentations who had debts to pay to continue coming to the practice for treatment despite lacking the means to pay for their health care. This kind of advocacy was common and seen as part of the responsibility of providers in the Pacific models of health earlier reviewed (Agnew et al., 2004). The Treaty of Waitangi principles aim to guide the health care delivery in Aotearoa New Zealand to ensure equity of uptake of services and satisfaction of the service user's healthcare experience as far as possible (Barnett \& Barnett, 2004; Barnett, Smith, \& Cumming, 2009).

\section{Relationship with community}

The establishment of PHOs was seen as a positive move by their managers as it provides an opportunity to collaborate to provide culturally appropriate services designed and delivered by Pacific clinicians. As one manager of a Pacific health organisation stated, it was envisaged that PHO funding would build capability in the workforce for care of Pacific by Pacific.

As services move into the community, we are organising the Pacific community to work as a team. Pacific people need to work together as a team that is how it works best. (Manager)

\section{Providing services in a shared language}

Another Pacific-led PHO used the services of a medical specialist to run a clinic to see patients who had been screened by a self-administered patient questionnaire to identify health issues. This consultant was unique in being able to speak a number of Pacific languages which enabled him to engage more easily with the majority of patients at that service. The shared language was an important means of building relationship with Pacific clients.

This is in contrast to comments made by a non-Pacific PHO about the difficulty of engaging with Pacific communities when the process was not relational (for example, one PHO mailed out about 5,000 letters to 
Pacific families and received less than 100 responses). This illustrates the importance of understanding how to engage Pacific and the value that Pacific practices bring in their capability to do this. Establishing processes and protocols for making decisions and acknowledging shared values, including the spiritual dimensions of care, aided success, as a $\mathrm{CEO}$ of a Pacific PHO explains:

We're bound by a common philosophy... I think fundamentally in essence we are a Christian organisation bound by a set of Christian values that hold us together in quite hard times and they are around all of those things, you know like..., integrity, respect... we do have hard times and we have our difficulties and battle but we try to work through them and there is a lot of passion. It's still trying to work through that respect and just wanting the best for our community. (CEO/Manager)

\section{Collaboration, co-ordination and team work across services}

Since their joining of a Pacific PHO, a common experience amongst participants was improved communication between diverse social and statutory agencies to avoid the silo-effect of services acting independently of one another. These social connections and networks enabled more comprehensive wrap-around services to be offered to Pacific patients.

The difference between [name of another PHO] and [name of participant's Pacific PHO] is the community focused, community driven, focus on, you know, the health needs of the people. Whereas [name of other PHO] is very much doctor driven now....(General practitioner)

Having a manager who shared a vision and philosophy of working with under-resourced communities was seen to be advantageous by colleagues working at the same PHO as a shared vision of the local community was facilitated. A common purpose for continuing to work within the $\mathrm{PHO}$ was a passion for work with what were considered to be under-resourced communities. As one participant commented:

Our manager [name] who is Māori understands where the lower socioeconomic people are coming from. She has a passion for this population here. And that's why we are getting that support because we know that she's there because of that passion. (Nurse)

Another participant who worked as a general practitioner in a not-for-profit $\mathrm{PHO}$ described this collaboration as "a collective approach to providing a service." This was seen by those interviewed as being part of this shared vision for work in the PHO:

We are not alone as [Pacific Islanders] within this PHO. We are here working alongside others and do collectively have a very strong communication strategy, making sure the population focus on their needs. (General practitioner)

\section{Barriers}

Initially there was enthusiasm about the funding available for services to improve access. There were many initiatives that participants considered were working effectively for people accessing the health care they required. However, high and complex patient needs inevitably increased the length of consultations which impacted on the workload of the PHOs' treatment providers such as general practitioners and nurses, as the following comment from a general practitioner working in a Pacific PHO illustrates:

The heavy workload is helping them [patients] with social issues, so, sickness benefit, housing, all immigration issues. There is a lot of expectation that we will help them with that. We do quite a lot of it which prolongs our consultation time with the doctor or nurse. There are social workers in public health that we pass things on to ... very nice to have social 
workers except that their contracts are all around youth ... But the strategy needs to cater for elderly and social issues a bit better. (General practitioner)

General practitioners working in Pacific-led PHOs found that they needed to take longer to explain medical screening procedures prior to undertaking them with Pacific patients. This work needed to be done in face-to-face mode as contact by telephone and letter did not work as effectively with Pacific patients. The unavailability of funded transport to treatment was seen as an obstacle by a clinical manager/general practitioner of one Pacific PHO:

We had a lot of DNAs [did not attends] and she [nurse] said to me yesterday that she thinks transport has got something to do with it and that if we could provide transport, that would really help.

(General practitioner)

Social problems were tackled by the nurses interviewed. For example, one nurse who had an established relationship with local social services organised food parcels from a local food bank for a patient who had not been eating an adequate diet due to lack of money to spend on grocery items. The lack of food had meant that he had become dizzy and fallen from scaffolding at work resulting in a trip to the local hospital's accident and emergency department. Through the PHO's nurse liaising with the accident and emergency department at the hospital, the reason for the accident was clarified with the patient and advocacy arranged with the social services.

\section{Discussion: The implications for Health Social Work}

Participants in the Pacific-led PHOs have suggested in this study the need to consider co-ordinated approaches to health care which are comprehensive, culturally appropriate and flexible to respond to local needs. These approaches derive from traditional Pacific beliefs which include "going the extra mile" to meet the consumer where they live in a diversity of local and cultural contexts. The importance of incorporating Pacific values and ways of being in primary health cannot be underestimated in the uptake of services (Agnew et al., 2004; Beddoe \& Deeney, 2012; Döbl et al., 2017; Pockett \& Beddoe, 2017). Previous studies provide evidence that community-based models of intervention contribute to positive health outcomes (Barwick, 2000; Beddoe \& Deeney, 2012; Döbl et al., 2017; Pockett \& Beddoe, 2017).

The service providers mentioned a number of Pacific models they drew from in their work that were used alongside clinical models of assessment and treatment. Many of these frameworks adopt a focus on wellness in the community and are underpinned by an ethos of altruism, interpersonal relationship and social inclusion. These same principles need also to guide the provision of secondary health services including social work in hospitals where the tasks involve returning clients to extended family in the community to support ongoing care.

Building trust and support at the first point of contact requires what has been termed "a roundabout Pacific rapport building approach" which is learned by healthcare providers in practice rather than in theory (Agnew et al., 2004, p. ix). This approach involves ensuring that patients feel comfortable in their surroundings as an integrated part of the health service delivery. Rapport building to engage patients and their families is considered an important requirement when working with Pacific peoples (Agnew et al., 2004). Pacific models and modes of service delivery are distinct from western models of care and remain implicit in the practices of the health care providers who use them. These styles of service delivery follow the principles underpinning the government's strategic direction for Pacific health care. These principles are: respecting Pacific culture; valuing family; quality health care, and working together (Minister of Health \& Minister of Pacific 
Island Affairs, 2010, p. 5). Social workers require a detailed understanding of Pacific principles and models in their tertiary training including both theory in the lecture room and practice in the field placement. Clinical supervision attending to cultural safety needs to be factored into the wider learning of social workers both before and after their courses of study, as a programme of ongoing professional development. The Aotearoa New Zealand Association of Social Workers would be well placed to attend to such professional development nationally with the support of the workforce (Beddoe \& Deeney, 2012; Döbl et al., 2017; Pockett \& Beddoe, 2017).

\section{Conclusion}

The results from this exploratory study reveal that the implementation of Pacific PHOs has provided a capability for better communication between various parties from board level down to those working at the community level. It has increased the cultural relevance of healthcare approaches offered by removing the economic imperative to manage health as a business. For example, providers in the Pacific-led PHOs offered a broader range of services including efforts in health promotion, advocacy and education in programmes. This development has meant expanded roles and responsibilities beyond the medical model. The expectation of work in these PHOs can sometimes test the reality for some of the providers interviewed and is clearly impacting on the recruitment and retention of staff as the role is less bounded and consultation times are longer due to the complexity of assessing social issues that inevitably impact health and wellbeing. The use of Pacific language was considered an important component of engaging successfully and working well with Pacific patients. Further research is needed to more clearly delineate what is uniquely Pacific in the approach of the Pacific PHOs.

Engagement in community is a core competency when practising social work within Pacific models of healthcare.

These competencies need to be reflected in the learning outcomes for social work programmes of education at undergraduate and graduate levels in Aotearoa New Zealand. Spirituality and a holistic approach are key aspects of Pacific models of healthcare involving collaboration and relationship at their core, which differ from more linear, expert-knows-best approaches. Ongoing professional development in Pacific models of healthcare and culturally based models of clinical supervision are areas for further research and development for social work and other healthcare providers in Aotearoa.

\section{Acknowledgement}

I wish to acknowledge the collaboration and guidance of the Pacific Health Team including the project leader, Dr Ausaga Faasalele Tanuvasa (Senior Research Fellow of the Health Services Research Centre of Victoria University of Wellington, New Zealand), Mili Burnette (Research Assistant, Health Services Research Centre), Dr Lanuola Asiasiga (Senior Researcher, Massey University) and Fuafiva Fa'alau (Contractor/Consultant) for their on-going advice and support. I also wish to thank the research participants for giving so generously of their time and expertise.

\section{References}

Agnew, F., Pulotu-Endemann, F. K., Robinson, G., Suaalii-Sauni, T., Warren, H., Wheeler, A.,... Schmidt-Sopoaga, H. (2004). Pacific models of mental health service delivery in New Zealand ("PMMHSD") project. Auckland, NZ: Waitemata District Health Board.

Anderson, I., Crengle, S., Kamaka M., Chen, T. H., Palafox, N., \& Jackson-Pulver, L. (2006). Indigenous health in Australia, New Zealand, and the Pacific. Lancet, 27, 367(9524), 1775-1185.

Barnett, R., \& Barnett, P. (2004). Primary healthcare in New Zealand: Problems and policy approaches. Social Policy Journal of New Zealand, 21, 49-66.

Barnett, P., Smith J., \& Cumming, J. (2009). The roles and functions of primary health organisations. Wellington, NZ: Health Services Research Centre, Victoria University of Wellington. Retrieved from https://www.victoria.ac.nz/ health/centres/health-services-research-centre/docs/ reports/Roles-and-Functions-of-PHOs.pdf

Barwick, H. (2000). Improving access to primary care for Māori and Pacific peoples: A literature review. Wellington, NZ: Health Funding Authority. 
Beddoe, L., \& Deeney, C. (2012). Discovering health social work in New Zealand in its published work: Implications for the profession. Aotearoa New Zealand Social Work, 24(1), 41-55.

Cumming, J., \& Mays, N. (2011). New Zealand's Primary Health Care Strategy: Early effects of the new financing and payment system for general practice and future challenges. Health Economics, Policy and Law, 6(1), 1-21. doi:10.1017/S1744133109990260

Cumming, J., Raymont A., Gribben B., Horsburgh M., Kent B., McDonald J.,... Smith, J. (2005). Evaluation of the implementation and intermediate outcomes of the Primary Health Care Strategy. Wellington, NZ: Health Services Research Centre, Victoria University of Wellington. Retrieved from https://www.victoria.ac.nz/ health/centres/health-services-research-centre/docs/ reports/downloads/FINAL-Overview-27-May.pdf

Döbl, S., Beddoe, L., \& Huggard, P. (2017). Primary health care social work in Aotearoa New Zealand: An exploratory investigation. Aotearoa New Zealand Social Work, 29(2), 119-130.

Gauld, R., \& Mays, N. (2006). Are New Zealand's new primary health organisations fit for purpose? British Medical Journal, 333, 1216-1218.

Hawley, N. L., \& McGarvey, S. T. (2015). Obesity and diabetes in Pacific Islanders: The current burden and the need for urgent action. Current Diabetes Reports, 15(5), 29-39. doi: 10.1007/s11892-015-0594-5.

Hogg, W., Rowan, M., Russell, G., Geneau, R., \& Muldoon, L. (2008). Framework for primary care organisations: The importance of a structural domain. International Journal for Quality in Healthcare, 20(5), 308-313. doi.org/10.1093/intqhc/mzm054

Jantrana, S., \& Crampton P. (2009). Primary health care in New Zealand: Who has access? Health Policy, 93(1), 1-10. doi:10.1016/j.healthpol.2009.05.006

Keating, G., \& Jaine, R. (2016). What supports are needed by New Zealand primary care to improve equity and quality? Journal of Primary Health Care, 8(4), 312-315. doi: $10.1071 / \mathrm{HC} 16020$

King, A. (2001). The primary health care strategy. Wellington, NZ: Ministry of Health.

Minister of Health \& Minister of Pacific Island Affairs. (2010). Ala Mo'ui': Pathways to Pacific health and well-being 2010-2014. Wellington, New Zealand: Author. Retrieved from https://www.health.govt.nz/system/files/documents/ publications/ala-moui-pathways-to-pacific-healthwellbeing2010-2014.pdf

Pack, M. (2008). An innovation in primary mental health services: The MidValley Well-being Service. Aotearoa New Zealand Social Work Review, 20(3), 25-33.

Pack, M., Minister, J., Churchward, M., \& Fa'asalele Tanuvasa, A. (2013). Evaluation of the implementation and immediate outcomes of the Primary Health Care Strategy: The experiences of Pacific PHOs and Pacific populations. Wellington, NZ: Health Services Research Centre, Victoria University of Wellington. Retrieved from https://www.victoria.ac.nz/health/centres/health-servicesresearch-centre/our-publications/reports/pacific-phcsreport.pdf

Pockett, R., \& Beddoe, L. (2017). Social work in health care: An international perspective. International Social Work, 60(1), 126-139.
Pulotu-Endemann, F. K. \& Faleafa, M. (2017). Developing a culturally competent workforce that meets the needs of Pacific people living in New Zealand. In M. Smith \& A. Jury (Eds.), Workforce development theory and practice in the mental health sector (pp. 165-180). doi:10.4018/978-1-5225-1874-7.ch008

Southwick, M., Kenealy, T., \& Ryan, D. (2012). Primary care for Pacific people: A Pacific and health systems approach. Report to the Health Research Council and the Ministry of Health. Retrieved from www.pacificperspective.co.nz

Statistics New Zealand. (2013). Quick Stats about culture and identity. Wellington, NZ: Author. Retrieved from http://archive.stats.govt.nz/Census/2013-census/profileand-summary-reports/quickstats-culture-identity/pacificpeoples.aspx

Suaalii-Sauni, T., Wheeler, A., Saafi, E., Robinson, G. Agnew, F., Warren, H., Erick, M., \& Hingano, T. (2009). Exploration of Pacific perspectives of Pacific models of mental health service delivery in New Zealand. Pacific Health Dialog, 15(1), 18-27.

Tamasese, K., Peteru, C., Waldegrave, C., \& Bush, A (2005). Ole taeao afua [the new morning]: A qualitative investigation into Samoan perspectives on mental health and culturally appropriate services. Australian and New Zealand Journal of Psychiatry, 39(4), 300-309.

Yin, R. (2009). Case study research: Design and methods (4th ed.). Thousand Oaks, CA: Sage. 